\title{
Collective Adoption of Max-Min Strategy in an Information Cascade Voting Experiment
}

\author{
Shintaro Mori ${ }^{1 *}$, Masato Hisakado ${ }^{2}$, and Taiki Takahashi ${ }^{3,4}$ \\ ${ }^{1}$ Department of Physics, Kitasato University \\ 1-15-1 Kitasato, Sagamihara, Kanagawa 252-0373, Japan \\ ${ }^{2}$ Standard and Poor's \\ 1-6-5 Marunouchi, Chiyoda-ku, Tokyo 100-0005, Japan \\ ${ }^{3}$ Department of Behavioral Science, Faculty of Letters \\ and \\ Center for Experimental Research in Social Sciences, Hokkaido University ${ }^{4}$ \\ Kita 10, Nishi 7, Kita-ku, Sapporo, Hokkaido 060-0810, Japan
}

\begin{abstract}
We consider a situation where one has to choose an option with multiplier $m$. The multiplier is inversely proportional to the number of people who have chosen the option and is proportional to the return if it is correct. If one does not know the correct option, we call him a herder, and then there is a zero-sum game between the herder and other people who have set the multiplier. The max-min strategy where one divides one's choice inversely proportional to $m$ is optimal from the viewpoint of the maximization of expected return. We call the optimal herder an analog herder. The system of analog herders takes the probability of correct choice to one for any value of the ratio of herders, $p<1$, in the thermodynamic limit if the accuracy of the choice of informed person $q$ is one. We study how herders choose by a voting experiment in which 50 to 60 subjects sequentially answer a two-choice quiz. We show that the probability of selecting a choice by the herders is inversely proportional to $m$ for $4 / 3 \leq m \leq 4$ and they collectively adopt the max-min strategy in that range.
\end{abstract}

KEYWORDS: herd, information cascade, zero-sum game, experiment, max-min strategy, econophysics, socio-physics

\section{Introduction}

Even if each person has limited information, aggregated information becomes very accurate. ${ }^{1}$ This is the wisdom of crowd effect, and is supported by many examples from political elections, sports predictions, quiz shows, and prediction markets. ${ }^{2-4}$ In contrast, in order to 
give accurate results, three conditions need to be satisfied: diversity, independence, and decentralization. If these conditions are not satisfied, aggregated information becomes unreliable or worse. ${ }^{2,5}$ However, in an ever-more connected world, it becomes more and more difficult to retain independence. Furthermore, if the actions or choices of others are visible, neglecting them is not realistic in light of the merit of social learning. ${ }^{6,7}$ In this case, information cascade may emerge and information aggregation ceases..$^{8-14}$

More concretely, we consider a situation where people sequentially answer a two-choice question with choices A and B. The payoff for the correct choice is constant . Before this question is asked, many other people have already answered and their choices are made known as $C_{A}$ people choosing A and $C_{B}$ people choosing $\mathrm{B}$, which is called social information. If the person answering knows the correct choice, he should choose it. His choice is not affected by social information. We then call him an independent voter. However, if he does not know the correct choice, he will be affected by social information. ${ }^{15} \mathrm{He}$ tends to go with the majority and we then call him a herder. By herding, the wisdom of crowds is on the edge. If a herder is isolated from others, his choice becomes A and B, and should be canceled. As a result, the choice by an independent voter remains. The majority choice always converges to the correct one in the limit of a large number of people. This is known as Condorcet's jury theorem. ${ }^{1}$ However, if others' choices are given as social information, the cancellation mechanism does not work. The herder copies the majority and ignores the correct information given by the independent voter. If the proportion of herders $p$ exceeds some threshold value $p_{c}$, there occurs a phase transition from the one-peak phase where the majority choice always converges to the correct one to the two-peak phase where the majority choice converges to the wrong one with a finite and positive probability. ${ }^{16}$ We call this phase transition the information cascade transition. ${ }^{16-18}$ This is the risk of imitation in the wisdom of crowd. How can we avoid this risk? There exists a hint in race-track betting markets ${ }^{19,20}$ and prediction markets. ${ }^{21,22} \mathrm{In}$ order to aggregate information scattered among people, the market mechanism can be very effective. ${ }^{2-4}$

We consider a situation in which each choice $\alpha \in\{A, B\}$ has a multiplier $M_{\alpha}$ that is inversely proportional to the number of subjects $C_{\alpha}$ who chose it. The payoff for the correct choice is proportional to the multiplier. If the multiplier of a choice is large, the number of people who chose it is small. If the return is constant, herder usually avoid the choice. However, now, the return on the correct choice is proportional to the multiplier, and hence we cannot say that herder does not choose it. Copying the majority gives him a small return, even if it is a correct choice. The multiplier plays the role of a "tax" on herding (free riding) and 
copying the minority can be an attractive choice. The situation is a zero-sum game between the herder answering and all the previous subjects who have set the multipliers. In zero-sum games, the max-min strategy maximizes the expected return and is optimal. ${ }^{23}$ In the above two-choice quiz, the max-min strategy is the one where a herder chooses $\alpha$ with a probability proportional to $C_{\alpha}$ and cancels the risk in expected return by the multipliers. We call the herder who adopts the optimal max-min strategy an analog herder. ${ }^{24,25}$ If a herder behaves as an analog herder, the convergence to equilibrium state becomes slow as $p$ increases and there occurs a phase transition in the convergence speed as $p$ exceeds half. ${ }^{25}$ However, the information cascade phase transition does not occur for $p<1$. A majority of people always choose the correct choice in the limit of a large number of people (thermodynamic limit) and the system is in the one-peak phase for any value of $p$ if the accuracy of the information of the independent voter $q$ is $q>1 / 2 .{ }^{25}$ Furthermore, the analog herder's choice does not affect the limit value of the percentage of correct answer and it converges to $q$. As for the two-choice quiz, the independent voter knows the correct choice and $q=1$ holds. In this case, the system of analog herders maximizes the probability of correct choice for $p<1$ in the thermodynamic limit. Even in limit $p \rightarrow 1$, the system can take the probability to one.

In this paper, we have adopted an experimental approach to study whether herders adopt the max-min strategy and behave as analog herders if the choices have multipliers. We have also studied a herder's probability of correct choice. The organization of the paper is as follows. We explain the experiment and derive the optimal max-min strategy in section 2 . The subjects answer a two-choice quiz in three cases $r \in\{O, C, M\}$. In case $O$, the subjects answer without social information. In cases $C$ and $M$, they receive social information based on previous subjects' choices. Social information is given as summary statistics $\left\{C_{A}, C_{B}\right\}$ in case $\mathrm{C}$ and as multipliers $\left\{M_{A}, M_{B}\right\}$ in case $\mathrm{M}$. Sections 3 and 4 are devoted to the analysis of the experimental data. In section 3, we summarize data about the macroscopic aspects of the system. In section 4, we derive a microscopic rule regarding how herders copy others in each case $r \in\{C, M\}$. In section 5, we introduce a stochastic model that simulates the system. We study the transition ratio $p_{c}(r)$ for cases $r \in\{C, M\}$. We estimate the probability of correct choice by the herders in the experiment and compare it with that of the optimal analog herders system. Section 6 is devoted to the summary and discussions. In the appendices, we give some supplementary information about the experiment and a simulation study of the convergence exponent. We also prove that only the system of analog herders can take the probability of correct choice to one in limit $p \rightarrow 1$. 


\section{Experimental setup and optimal strategy in case $M$}

\subsection{Experimental setup}

The experiment reported here was conducted at the Group Experiment Laboratory of the Center for Experimental Research in Social Sciences at Hokkaido University. We have conducted two experiments. We call them EXP-I and EXP-II. In EXP-I (II), we recruited 120 (104) students from the university. We divided them into two groups, Group A and Group B, and prepared two sequences of subjects of average length 60 (52). The main motive to divide the subjects into two groups is to ensure many choice sequences in order to estimate the average value of macroscopic quantities. In addition, we can check the estimation of herders' ratio $p$ by comparing the values from the two groups for the same question. ${ }^{16}$

The subjects sequentially answered a two-choice quiz of 120 questions. Some subjects could not answer all the questions within the alloted time, and so the number $T$ of subjects who answered a particular question varied. We label the questions by $i \in\{1,2, \cdots, 120\}$ and denote the length of the sequence of the subjects for question $i$ by $T_{i}$. In EXP-I, the subject answers in three cases $r \in\{O, C, M\}$ in this order. We denote the answer to question $i$ in case $r$ after $t-1$ subjects' answers by $X(i, t \mid r)$, which takes the value $1(0)$ if the choice is true (false). The order $t$ of the subject in the choice sequence $\{X(i, t \mid r)\}$ plays the role of time. $\left\{C_{0}(i, t \mid r), C_{1}(i, t \mid r)\right\}$ are the number of subjects who choose true and false for question $i$ among the prior $t$ subjects and are given as

$$
\begin{aligned}
& C_{1}(i, t \mid r)=\sum_{t^{\prime}=1}^{t} X\left(i, t^{\prime} \mid r\right), \\
& C_{0}(i, t \mid r)=t-C_{1}(i, t \mid r) .
\end{aligned}
$$

In case $O$, the subject answered without any social information. Then, he answered in case $C$. When $t-1$ subjects have already answered question $i$ before him in his group, he received summary statistics $\left\{C_{0}(i, t-1 \mid C), C_{1}(i, t-1 \mid C)\right\}$ from all of them. For the correct choice in cases $O$ and $C$, the subject gets two points. Finally, in case $M$, when $t-1$ subjects have already answered question $i$ before him in his group, the subject receives multipliers $\left\{M_{0}(i, t-\right.$ 1), $\left.M_{1}(i, t-1)\right\}$ from all previous $t-1$ subjects. For the correct choice, the subject gets the points which is given by the multiplier. The multiplier $M_{\alpha}$ for $\alpha \in\{0,1\}$ was calculated based on the summary statistics in case $M$ as

$$
\begin{aligned}
M_{\alpha}(i, t-1) & =\frac{C_{0}(i, t-1 \mid M)+C_{1}(i, t-1 \mid M)+1}{C_{\alpha}(i, t-1 \mid M)+1} \\
& =\frac{t}{C_{\alpha}(i, t-1 \mid M)+1} .
\end{aligned}
$$


The multiplier is given by dividing total points $C_{0}+C_{1}+1=t$ for all subjects with choice value among $C_{\alpha}+1$ subjects who have chosen $\alpha$. This is similar to the payoff odds of the parimutuel system in gambling.

In EXP-II, in addition to the three cases $r \in\{O, C, M\}$, the subjects answered in at most four cases $r \in\{1,5,11,21\}$ between cases $O$ and $C$. In cases $r \in\{1,5,11,21\}$, the subject received summary statistics $\left\{C_{0}(i, t-1 \mid r), C_{1}(i, t-1 \mid r)\right\}$ from previous $r$ subjects. $C_{0}(i, t-$ $1 \mid r)+C_{1}(i, t-1 \mid r)=r$ holds and as $r$ increases, the amount of social information increases. In EXP-I, the amount of social information increases rapidly from $r=0$ in case $O$ to $r=t-1$ in case $C$. In EXP-II, $r$ gradually increases. The payoff for the correct choice is 1 in cases $r \in\{O, 1,5,11,21, C\}$ and the multiplier in case $M$. Detailed information about EXP-II has been presented in our previous work, ${ }^{16}$ where we have studied the experimental data for cases $r \in\{O, 1,5,11,21, C\}$. In this paper, we concentrate on case $M$ and take case $C$ as the control case.

We repeated the same experiment for both Groups A and B. We obtained $120 \times 2$ sequences $\{X(i, t \mid r)\}$ for each $r \in\{O, C, M\}$. We label the sequence in Group B by $i+120$, so that $i \in\{1,2, \cdots, 240\}$. The experimental design is summarized in Table I.

Table I. Experimental design. $T$ means the number of subjects and $\{r\}$ means the cases where the subjects answered the quiz. I means the number of questions. The length $T_{i}$ of sequence $\{X(i, t \mid r)\}$ for question $i$ is almost the same as $T$ in EXP-I. In EXP-II, it depends on $i$ and the average value is 50.8 .

\begin{tabular}{lcccc}
\hline Experiment & Group & $T$ & Cases $\{r\}$ & $I$ \\
\hline EXP-I & A & 57 & $\{O, C, M\}$ & 120 \\
EXP-I & B & 63 & $\{O, C, M\}$ & 120 \\
EXP-II & A & 52 & $\{O, 1,5,11,21, C, M\}$ & 120 \\
EXP-II & B & 52 & $\{O, 1,5,11,21, C, M\}$ & 120 \\
\hline
\end{tabular}

\subsection{Max-Min Strategy in case M}

We derive the optimal strategy for herders in case $M$. A subject can choose $\alpha \in\{A, B\}$. We suppose that he votes one unit for a choice and call him a voter. Here, we consider the case where one vote can be divided by the voter. If a voter believes $A$ is correct, he votes one unit for $A$. If the voter does not know the answer at all, he votes 0.5 unit for $A$ and 0.5 unit for $B$. We assume that a voter thinks the probability that $A$ is correct is $\beta$, and the probability that $B$ is correct is $1-\beta$. The voter divides one unit vote into $x$ for $A$ and $1-x$ for $B$ by his 
decision making. Expected return $R$ is

$$
\begin{aligned}
R & =\beta \cdot M_{A} \cdot x+(1-\beta) \cdot M_{B} \cdot(1-x) \\
& =\beta\left(M_{A} x-M_{B}(1-x)\right)+M_{B}(1-x) .
\end{aligned}
$$

We assume that herders do not have information about the correct answers without multipliers $\left\{M_{A}, M_{B}\right\}$. Hence, we assume that a herder cannot estimate the probabilities of correct answer $\beta$ as Knightian uncertainty, because he has no knowledge to answer the question. ${ }^{26}$ The situation is a zero-sum game between the herder and other previous voters as the multipliers are set such that all votes are divided by the voters who have chosen the correct option. The max-min strategy has been proved to be optimal in game theory. ${ }^{23}$ The voter minimizes the expected loss due to the uncertainty in the choice. In order to minimize the expected loss from the uncertainty, it should be chosen such that $M_{A} \cdot x=M_{B} \cdot(1-x)$ holds, from (1). This position has no sensibility for $\beta$.

We can calculate $x$ from (1),

$$
x=\frac{M_{B}}{M_{B}+M_{A}} .
$$

As multiplier $M_{\alpha}$ is calculated as

$$
M_{\alpha}=\frac{t+1}{C_{\alpha}+1}
$$

ratio $x$ for $A$ is then

$$
x=\frac{C_{A}+1}{t+2} \sim \frac{C_{A}}{t} \text { for } t>>1 .
$$

$x$ becomes proportional to $C_{A}$ and it is the voting strategy of analog herders. ${ }^{25}$

The discussion shows that the strategy of analog herders is optimal for a herder as it maximizes his expected return. In our experiment, the voter cannot divide one's vote (choice). Hence, the averaged behavior of herders becomes akin to that of the analog herders, when herders adopt the optimal strategy.

We make one comment about the optimal strategy for the independent voter. When $\beta=1$, the voter believes his information and chooses what he believes to be true. When $\beta<1$, it is not optimal to do so in general. The expected return $R$ in (1) is

$$
R=\left(\beta \cdot M_{A}-(1-\beta) \cdot M_{B}\right) x+M_{B}(1-\beta) .
$$

By maximizing $R$, we obtain $x$ as

$$
x=\theta\left(\beta \cdot M_{A}-(1-\beta) \cdot M_{B}\right) .
$$

Here, $\theta$ is a Heaviside (step) function. If $\beta \cdot M_{A}>(1-\beta) \cdot M_{B}$, he chooses $A$ and vice versa. 
He behaves as an "arbitrager" for $\beta<1$. It is the risk-neutral strategy that has been discussed in the context of racetrack betting markets and prediction markets. ${ }^{20,22}$

\section{Data analysis : Macroscopic Aspects}

We obtained 240 sequences $\{X(i, t \mid r)\}, t \in\left\{1,2, \cdots, T_{i}\right\}$ for question $i \in\{1, \cdots, 240\}$ and cases $r \in\{O, C, M\}$ in each experiment. Data $\{X(i, t \mid r)\}$ for both experiments is downloadable at http://arxiv.org/abs/1211.3193. The percentage of correct answers of sequence $\{X(i, t \mid r)\}$ for question $i$ in case $r$ is defined as $Z(i \mid r)=\sum_{s=1}^{T_{i}} X(i, s \mid r) / T_{i}$. In the analysis, the subjects are classified into two categories-independent voters and herders-for each question. We assume that the probability $q$ of a correct choice for independent voters and herders is $100 \%$ and $50 \%$, respectively. ${ }^{16}$ For a group with $p(i)$ herders and $1-p(i)$ independent voters, the expectation value of $Z(i \mid O)$ is $1-p(i) / 2$. The maximal likelihood estimate of $p(i)$ is given as $p(i)=$ $2(1-Z(i \mid O))$.

\subsection{Distribution of $Z(i \mid r)$}

There are 240 samples of sequences of choices for each $r$. We divide these samples into 11 bins according to the size of $Z(i \mid r)$, as shown in Table II. The number of data samples in each bin for cases $r \in\{O, C, M\}$ are given in the second, third and fourth column as $N($ No. $\mid r)$. Social information causes remarkable changes in subjects' choices. For case $O$, there is one peak at No. 7, and for case $C(M)$, there are peaks at No. 2 (4) and No. 11 (10) in EXP-I. The samples in each bin of case $O$ share almost the same value of $p$. For example, in the samples of No. 6 bin $(0.45<Z(i \mid O) \leq 0.55)$, there are almost only herders in the subjects' sequence and $p(i) \simeq 100 \%$. In contrast, in the samples of No. 11 bin $(Z(i \mid O)>0.95)$, almost all subjects know the answer to the questions and are independent $(p(i) \simeq 0 \%)$. An extremely small value of $Z(i \mid O)$ indicates some bias in the question and we omit the samples that satisfy $Z(i \mid O)<0.45$. In addition, the minimum value of $Z(i \mid r)$ should be $1-p(i)$. If $Z(i \mid r)<1-p(i)$, it means that the estimation of $p(i)$ for the sequence $\{X(i, t \mid r)\}$ fails. The true value of $p(i)$ should be larger than the estimated value. We cannot give the appropriate estimation of $p(i)$ for the choice sequence and we omit the samples that satisfy $Z(i \mid C)<1-p(i)$ or $Z(i \mid M)<1-p(i)$. From these procedures, we are left with 167 (177) samples in EXP-I (II) and we denote the set by $I^{\prime}$. $I$ (No.) denotes the set of samples in each bin in case $O$ among $I^{\prime}$.

We comment about the above data elimination procedure. The main purpose of the experiment is to clarify how herders copy others' choices. For the purpose, it is necessary to assure that herders choose each option with equal probability in case $O$ and the herder's $q$ is 
Table II. Effect of social information on subjects' decisions. The upper (lower) table summarizes the data for EXP-I (II). We divide the samples according to the size of $Z(i \mid r)$. $N($ No. $\mid r)$ denotes the number of samples for case $r$ in each bin. $I$ (No.) is the set of sample $i$ in each bin of case $O$ after removing the samples that satisfy $Z(i \mid O)<45 \%$ or $Z(i \mid C)<(1-p(i))$ or $Z(i \mid M)<(1-p(i))$. $\mid I($ No. $) \mid$ means the number of samples in the set. $p_{\text {avg }}$ is estimated as the average value of $p(i)=2(1-Z(i \mid O))$ over the samples in $I$ (No.). In the last two columns, the ratio of the case with $\{Z(i \mid r)<1 / 2\}$ for $r \in\{C, M\}$ among the samples in $I$ (No.) is shown.

\begin{tabular}{rcccccccc} 
No. & $Z(i \mid r)[\%]$ & $N($ No. $\mid O)$ & $N($ No. $\mid C)$ & $N($ No. $\mid M)$ & $\mid I($ No. $) \mid$ & $p_{\text {avg }}($ No. $)[\%]$ & $Z(i \mid C)<1 / 2$ & $Z(i \mid M)<1 / 2$ \\
\hline 1 & $<5$ & 0 & 5 & 0 & NA & NA & NA & NA \\
2 & $5 \sim 15$ & 3 & 33 & 7 & NA & NA & NA & NA \\
3 & $15 \sim 25$ & 5 & 28 & 25 & NA & NA & NA & NA \\
4 & $25 \sim 35$ & 18 & 9 & 30 & NA & NA & NA & NA \\
5 & $35 \sim 45$ & 35 & 5 & 13 & NA & NA & NA & NA \\
6 & $45 \sim 55$ & 38 & 5 & 13 & 38 & 97.5 & $18 / 38$ & $17 / 38$ \\
7 & $55 \sim 65$ & 57 & 5 & 14 & 52 & 78.3 & $7 / 52$ & $5 / 52$ \\
8 & $65 \sim 75$ & 29 & 7 & 19 & 26 & 60.3 & $0 / 26$ & $0 / 26$ \\
9 & $75 \sim 85$ & 41 & 17 & 44 & 38 & 40.6 & $0 / 38$ & $0 / 38$ \\
10 & $85 \sim 95$ & 11 & 57 & 62 & 11 & 21.3 & $0 / 11$ & $0 / 11$ \\
11 & $\geq 95$ & 3 & 69 & 13 & 2 & 5.1 & $0 / 2$ & $0 / 2$ \\
\hline Total & & 240 & 240 & 240 & 167 & $66.8 \%$ & $25 / 167$ & $22 / 167$
\end{tabular}

\begin{tabular}{rcccccccc} 
No. & $Z(i \mid r)[\%]$ & $N($ No. $\mid O)$ & $N($ No. $\mid C)$ & $N($ No. $\mid M)$ & $\mid I($ No. $) \mid$ & $p_{\text {avg }}($ No. $)[\%]$ & $Z(i \mid C)<1 / 2$ & $Z(i \mid M)<1 / 2$ \\
\hline 1 & $<5$ & 0 & 2 & 0 & NA & NA & NA & NA \\
2 & $5 \sim 15$ & 0 & 18 & 6 & NA & NA & NA & NA \\
3 & $15 \sim 25$ & 8 & 22 & 18 & NA & NA & NA & NA \\
4 & $25 \sim 35$ & 16 & 20 & 23 & NA & NA & NA & NA \\
5 & $35 \sim 45$ & 36 & 8 & 16 & NA & NA & NA & NA \\
6 & $45 \sim 55$ & 43 & 9 & 19 & 43 & 96.7 & $16 / 43$ & $15 / 43$ \\
7 & $55 \sim 65$ & 46 & 10 & 16 & 45 & 79.3 & $8 / 45$ & $3 / 45$ \\
8 & $65 \sim 75$ & 45 & 14 & 26 & 45 & 62.7 & $2 / 45$ & $0 / 45$ \\
9 & $75 \sim 85$ & 33 & 33 & 56 & 33 & 41.9 & $0 / 33$ & $0 / 33$ \\
10 & $85 \sim 95$ & 11 & 67 & 54 & 11 & 21.3 & $0 / 11$ & $0 / 11$ \\
11 & $\geq 95$ & 2 & 37 & 6 & 0 & NA & NA & NA \\
\hline Total & & 240 & 240 & 240 & 177 & $68.7 \%$ & $26 / 177$ & $18 / 177$
\end{tabular}

$50 \%$. This is the precondition of the experiment. We assume $q=0.5$ and derive the above three conditions that $Z(i \mid r)$ should satisfy. If $Z(i \mid r)$ contradicts with at least one of the conditions, there is some bias in the options. The data for question $i$ does not meet the precondition and we discard it in the analysis of the experimental data. The elimination procedure cannot 
assure the precondition with absolute certainty, it is indispensable.

We calculate the average value of $p(i)$ for the samples in $I$ (No.). We denote it as $p_{\text {avg }}$ (No.) and estimate it as

$$
p_{\text {avg }}(\text { No. })=\frac{1}{|I(\mathrm{No} .)|} \sum_{i \in I(\text { No. })} p(i) .
$$

Here, $\mid I($ No. $) \mid$ in the denominator means the number of samples in $I$ (No.), which is given in the sixth column of the table. In the last two columns, we show the ratio of the case with $\{Z(i \mid r)<1 / 2\}$ for $r \in\{C, M\}$ among the samples in $I($ No. $\mid O)$. In both cases, as $p_{\text {avg }}$ increases, the ratio increases rapidly to about half.
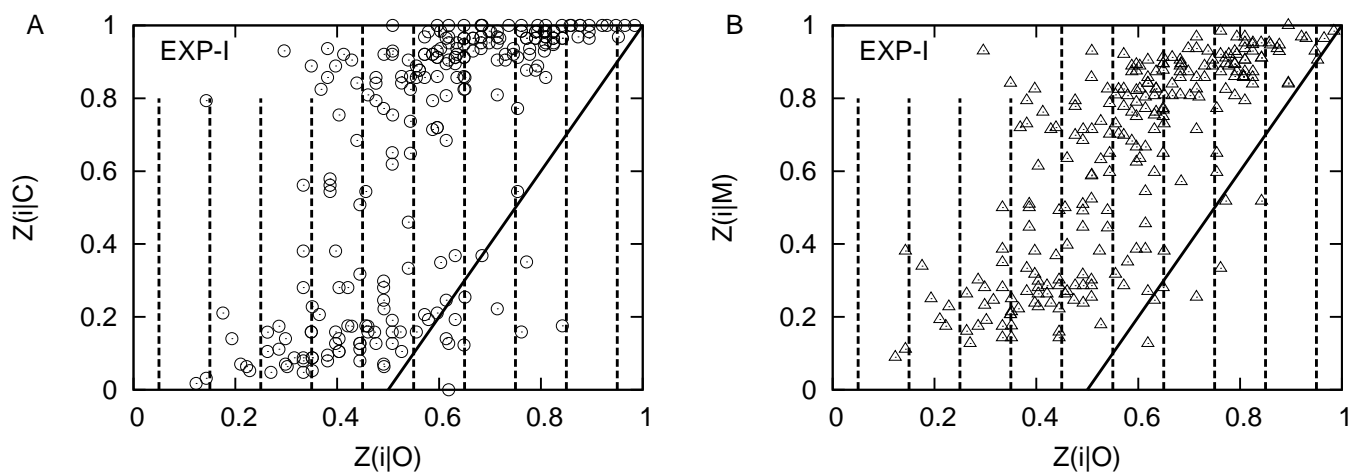

Fig. 1. Scatter plots of $Z(i \mid O)$ vs. $Z(i \mid r)$ for (A) Case $C$ and (B) Case $M$. The vertical lines show the border of the bins in Table II. The rising diagonal line from $(0.5,0)$ to top right shows the boundary condition $Z(i \mid r)=1-p$.

In order to see the social influence more pictorially, we show the scatter plots of $Z(i \mid O)$ vs. $Z(i \mid r), r \in\{C, M\}$ of EXP-I in Fig. 1. The $x$-axis shows $Z(i \mid O)$ and the $y$-axis shows $Z(i \mid r)$. The vertical lines show the boundary between the bins (from No. 1 to No. 11) for case $O$ in Table II. The rising diagonal line from $(0.5,0)$ to top right shows the boundary condition $Z(i \mid r)=1-p$. If subjects' answers are not affected by social information, data would distribute on the diagonal line from $(0,0)$ to top right. As the plots clearly indicate, the samples scatter more widely in the plane in case $C$ than in case $M$, which means that social influence is bigger in case $C$. For the samples with $Z(i \mid O) \geq 0.65$ in case $O$ (Nos. 8, 9, 10, and 11 bins in Table II), the changes, $Z(i \mid C)-Z(i \mid O)$, are almost positive and $Z(i \mid C)$ takes a value of about 1 in case $C$. In case $M$, the changes, $Z(i \mid M)-Z(i \mid O)$, are also almost positive and $Z(i \mid M)$ takes a value of about 0.9 . The average probability of choosing the correct option improves with social information for the samples in both cases. In contrast, for the samples with $0.45 \leq Z(i \mid O)<0.65$ (Nos. 6 and 7 bins in Table II), social information does not 
necessarily improve average performance. There are many samples with $Z(i \mid r)-Z(i \mid O)<0$ in both cases. These samples constitute the lower peak in Table II.

\subsection{Asymptotic behavior of the convergence}

We have seen drastic changes in the distribution of $Z(i \mid r)$ from the distribution of $Z(i \mid O)$. Table II and Figure 1 show the two-peak structure in the distribution of $Z(i \mid r)$. In our previous work on the information cascade phase transition, ${ }^{16}$ we have studied the time dependence of the convergence behavior of the sequences $\{X(i, t \mid r)\}$.

We denote the ratio of correct answers, $\frac{\left.C_{1}(i, t) r\right)}{t}$, as

$$
Z(i, t \mid r) \equiv \frac{C_{1}(i, t \mid r)}{t}=\frac{1}{t} \sum_{s=1}^{t} X(i, s \mid r)
$$

$Z\left(i, T_{i} \mid r\right)=Z(i \mid r)$ holds by definition. By studying the asymptotic behavior of the convergence of sequence $\{Z(i, t \mid r)\}$ for the samples in $I$ (No.), one can clarify the possibility of the information cascade transition by varying $p$. The variance of $Z(i, t \mid r)$ for the samples in $I$ (No.) is defined as

$$
\begin{aligned}
& \operatorname{Var}(Z(i, t \mid r)) \text { No. } \\
= & \frac{1}{\mid I(\text { No. }) \mid} \sum_{i \in I(\text { No. })}\left(Z(i, t \mid r)-<Z(i, t \mid r)>{ }_{\text {No. }}\right)^{2} \\
& <Z(i, t \mid r)>_{\text {No. }}=\frac{1}{\mid I(\text { No. }) \mid} \sum_{i \in I(\text { No. })} Z(i, t \mid r) .
\end{aligned}
$$

Here, we denote the average value of $Z(i, t \mid r)$ over the samples in $I($ No. $)$ by $\left\langle Z(i, t \mid r)>_{\text {No. }}\right.$. In the one-peak phase, the variance of $Z(i, t \mid r)$ for the samples with the same $p$ converges to zero in thermodynamic limit $t \rightarrow \infty$. In the analysis of experimental data, the values of $p$ have some variance among the samples in each bin, and $\operatorname{Var}(Z(i, t \mid r))$ No. takes small values in the limit. Depending on the convergence behavior, the one-peak phase is classified into two phases. ${ }^{18}$ If $\operatorname{Var}(Z(i, t \mid r))_{\text {No. }}$ shows normal diffusive behavior as $\operatorname{Var}\left((Z(i, t \mid r))_{\text {No. }} \propto t^{-1}\right.$, it is called the normal diffusion phase. We note that the variance is estimated for the ratio, $C_{1}(i, t \mid r) / t$, and the usual behavior $t^{1}$ for the sum of $t$ random variables is replaced by $\propto t / t^{2}=$ $t^{-1}$. If convergence is slow and $\operatorname{Var}(Z(i, t \mid r))_{\text {No. }} \propto t^{-\gamma}$ with $0<\gamma<1$, it is called the super diffusion phase. ${ }^{27}$ In the two-peak phase, $\operatorname{Var}(Z(i, t \mid r))_{\text {No. }}$ converges to some finite value in limit $t \rightarrow \infty .{ }^{17}$

Figure 2 shows the double logarithmic plots of $\operatorname{Var}(Z(i, t \mid r))_{\text {No. }}$ as a function of $t$. We see that convergence becomes very slow as $p_{\text {avg }}($ No. $)$ increases in general. The convergence exponent $\gamma$ is estimated by fitting with $\propto t^{-\gamma}$ for $t \geq 10$ in EXP-I. It decreases almost mono- 

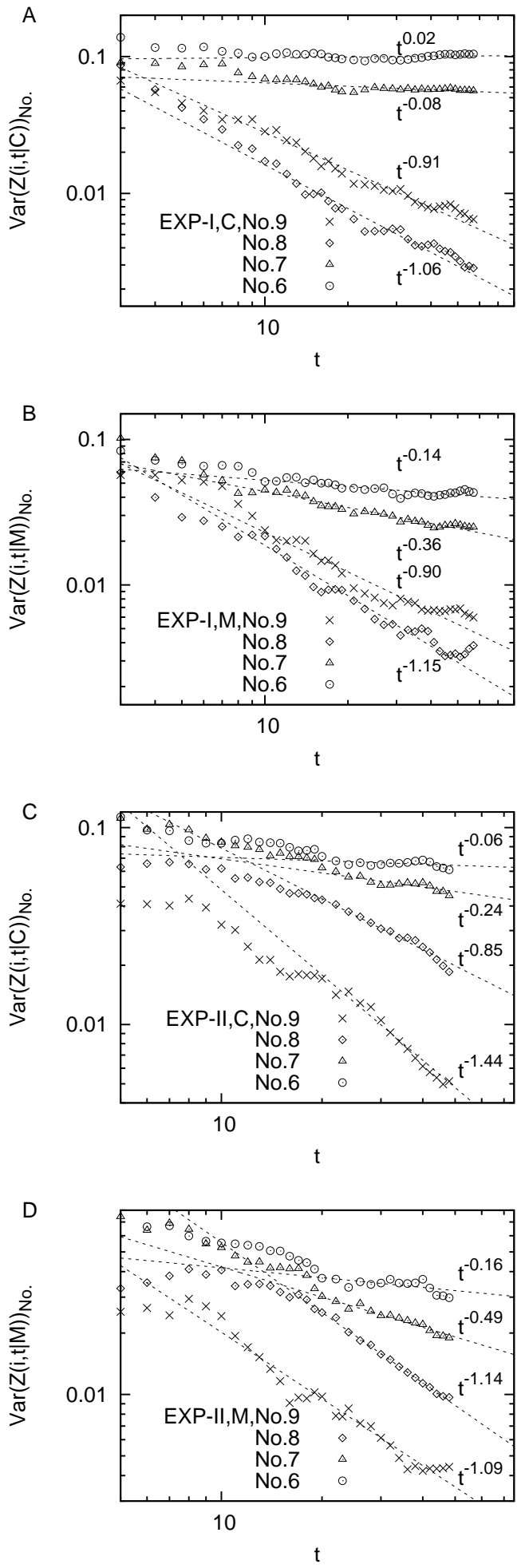

Fig. 2. Convergent behavior. Convergence is given by the double logarithmic plot of $\operatorname{Var}(Z(i, t \mid r))_{\text {No. }}$ vs. $t$ using the samples in four bins (Nos. $6(\circ), 7(\triangle), 8(\diamond)$, and $9(\times)$ in Table II) for (A) Case $C$ in EXP-I, (B) Case $M$ in EXP-I, (C) Case $C$ in EXP-II, and (D) Case $M$ in EXP-II. The dotted lines are fitted results with $\propto t^{-\gamma}$ for $t \geq 10(20)$ in EXP-I (II). 
tonically from about 1 to $-0.02(0.14)$ with an increase in $p_{\text {avg }}$ in case $C(M)$. Taking into account the estimate error of the exponent given in Appendix E,

$\gamma_{\mathrm{s}}$ are almost 1 for the samples in $I(9)$ and $I(8)$, and the system is in the normal diffusion (one-peak) phase in both cases $r \in\{C, M\}$. For the samples in $I(7), \gamma \mathrm{s}$ are apparently smaller than 1 and the system might be in the super diffusion phase. For the samples in $I(6)$, $\gamma$ becomes negative $(\gamma=-0.02)$ in case $C$. This suggests that the system is in the two-peak phase for the samples in $I(6) .{ }^{16}$ In case $M, \gamma$ is positive even for the samples in $I(6)$ and the system might be in the super diffusion phase. However, the result does not necessarily deny the existence of the two-peak phase, taking into account the variance of $p(i)$ and the estimate error of $\gamma$ from the limited sample size. We can only say that if the two-peak phase exists, the threshold value $p_{c}$ in case $M$ is considerably larger than that in case $C$.

\section{Data analysis: Microscopic Aspects}

In this section, we study the microscopic aspects of the herders. We clarify how they copy others' choices and derive a microscopic rule in each case $r \in\{C, M\}$. In particular, we study whether they behave as analog herders in case $M$.

\subsection{How do herders copy others?}

We determine how a herder's decision depends on social information. For this purpose, we need to subtract independent subjects' contribution from $X(i, t+1 \mid r)$. The probability of being independent is $1-p(i)$, and such a subject always chooses 1 . A herder's contribution is estimated as

$$
(X(i, t+1 \mid r)-(1-p(i))) / p(i)
$$

How the herder's decision depends on $C_{1}(i, t \mid r)=n_{1}$ is estimated by the expectation value of $(X(i, t+1 \mid r)-(1-p(i)) / p(i)$ under this condition. The expectation value means the probability that a herder chooses an option under the influence of prior $n_{1}$ subjects among $t$ who choose the same option. We denote it by $q_{h}\left(t, n_{1} \mid r\right)$, and estimate it as

$$
q_{h}\left(t, n_{1} \mid r\right)=\frac{\sum_{i \in I^{\prime}}\left[\frac{X(i, t+1 \mid r)-(1-p(i))}{p(i)}\right] \delta_{C_{1}(i, t \mid r), n_{1}}}{\sum_{i \in I^{\prime}} \delta_{C_{1}(i, t \mid r), n_{1}}} .
$$

Here, $\delta_{i, j}$ is $1(0)$ if $i=j(i \neq j)$ and the denominator is the number of sequences where $C_{1}(i, t \mid r)=n_{1}$. From the symmetry between $1 \leftrightarrow 0$, we assume that $q_{h}\left(t, n_{1} \mid r\right)=1-q_{h}(t, t-$ $\left.n_{1} \mid r\right)$. We study the dependence of $q_{h}\left(t, n_{1} \mid r\right)$ on $n_{1} / t$ and round $n_{1} / t$ to the nearest values in $\{k / 13(12) \mid k \in\{0,1,2, \cdots, 13(12)\}\}$ in EXP-I (II).

Figure 3 shows the plot of $q_{h}\left(t, n_{1} \mid r\right)$ for (A) case $C$ and (B) case $M$. We can clearly see 

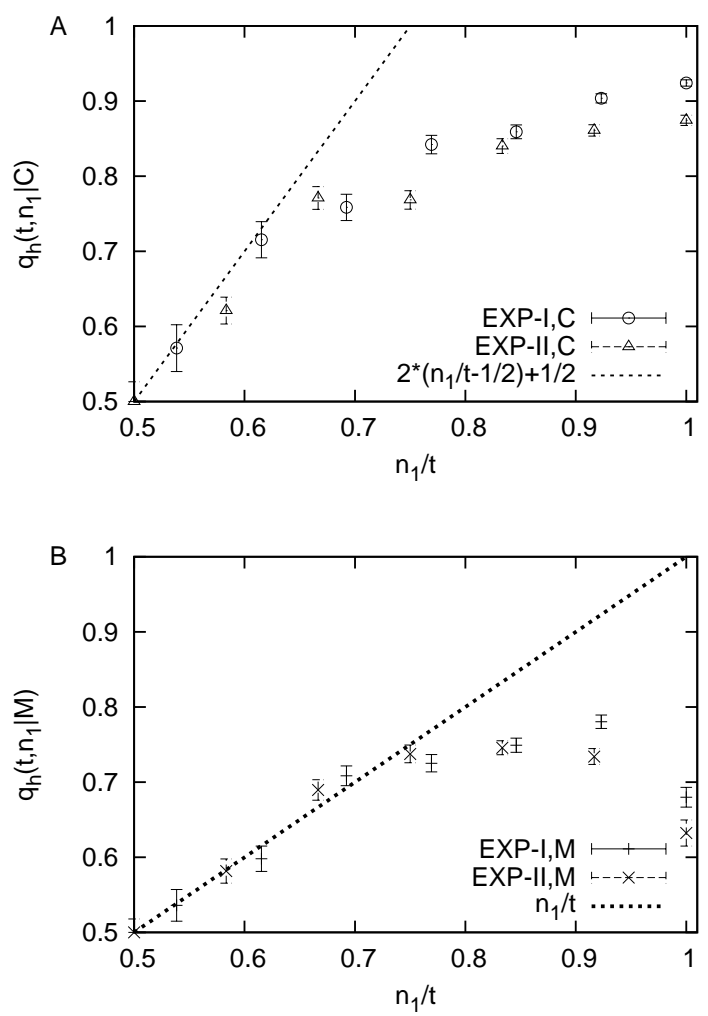

Fig. 3. Microscopic rule of herder's decision for (A) Case $C$ and (B) Case $M$. It shows the probability $q_{h}\left(t, n_{1} \mid r\right)$ that a herder chooses an option under the influence of prior $n_{1}$ subjects among $t$ choosing that option in case $r$. The thin dashed line in (A) shows $2\left(n_{1} / t-1 / 2\right)+1 / 2$. The dotted diagonal line in (B) shows the analog herder model $q_{h}\left(t, n_{1}\right)=n_{1} / t$.

the strong tendency to copy others in case $C$. As $n_{1} / t$ increases from $1 / 2, q_{h}\left(t, n_{1} \mid C\right)$ rapidly increases and the slope at $n_{1} / t=1 / 2$ is about 2.0 in EXP-I. Such nonlinear behavior is known as a quorum response in social science and ethology. ${ }^{28}$ The magnitude of the slope measures the strength of the herders' response. Comparing EXP-I and EXP-II, the response of herders is more sharp in EXP-I than in EXP-II. In EXP-II, where the amount of social information increases gradually, the subjects tend to copy others' choices more prudently than in EXP-I. If the slope exceeds 1 , the system shows the information cascade transition. The transition ratio $p_{c}$ depends on the slope. In the digital herders case, where $q_{h}\left(t, n_{1}\right)=\theta\left(n_{1}-t / 2\right)$ and the slope is infinite, $p_{c}$ takes $0.5 .{ }^{17}$ As the slope reduces to $1, p_{c}$ increases to 1 and the phase transition disappears in the limit. ${ }^{25}$

Contrary to case $C$, the dependence of $q_{h}\left(t, n_{1} \mid M\right)$ on $n_{1} / t$ is weak and the slope at $n_{1} / t=$ $1 / 2$ is almost 1 in case $M$. In range $1 / 4 \leq n_{1} / t \leq 3 / 4, q_{h}\left(t, n_{1} \mid M\right)$ lies on the diagonal dotted line and the herders almost behave as analog herders. As the multiplier $m$ is the inverse 
of $n_{1} / t$ for a large $t$, the average herder adopts the optimal max-min strategy in the range $4 / 3 \leq m \leq 4$. As the slope at $n_{1} / t=1 / 2$ is small, if the information cascade phase transition occurs, the transition ratio $p_{c}$ should become large as compared to in case $C$. One can also see an interesting behavior of herders. If the minority choice ratio $n_{1} / t$ is smaller than $1 / 4$ and multiplier $m$ exceeds 4 , some herders make the choice. As a result, if $n_{1} / t>3 / 4, q_{h}\left(t, n_{1} \mid M\right)$ becomes almost constant, about 3/4. We can interpret this as some of the herders preferring a big multiplier (long-shot) and $q_{h}\left(t, n_{1}\right)$ saturating at $3 / 4$.

\section{Analysis with stochastic model}

In this section, we simulate the system by a stochastic model, which we call a voting model. We consider a system with $p$ herders and $1-p$ independent voters. We estimate the transition ratio $p_{c}$ and herder's probability of correct choice in the experiment and compared it with that for the analog herders system.

\subsection{Voting model and thermodynamic limit}

We introduce a stochastic process $\{X(t \mid p)\}, t \in\{1,2,3, \cdots, T\}$ for $p \in[0,1] . X(t+1 \mid p) \in$ $\{0,1\}$ is a Bernoulli random variable. Its probabilistic rule depends on $C_{1}(t)=\sum_{t^{\prime}=1}^{t} X\left(t^{\prime} \mid r, p\right)$ and herders' proportion $p$. Given $\left\{C_{1}(t)=n_{1}\right\}$, we denote the probability that a herder chooses (copies) the correct option by $q_{h}\left(t, n_{1}\right)$. As $q_{h}\left(t, n_{1}\right)$ has symmetry $q_{h}\left(t, n_{1}\right)=1-q_{h}\left(t, t-n_{1}\right)$, $q_{h}\left(t, n_{1}\right)$ takes $1 / 2$ at $n_{1} / t=1 / 2$. We assume that $q_{h}\left(t, n_{1}\right)$ is a smooth and monotonically increasing function of $n_{1} / t$. The probabilistic rule that $X(t+1 \mid r, p)$ obeys under the condition is

$$
\begin{aligned}
& \operatorname{Prob}\left(X(t+1 \mid p)=1 \mid n_{1}\right)=(1-p)+p \cdot q_{h}\left(t, n_{1}\right), \\
& \operatorname{Prob}\left(X(t+1 \mid p)=0 \mid n_{1}\right)=p \cdot\left(1-q_{h}\left(t, n_{1}\right)\right) .
\end{aligned}
$$

We denote the probability that $X(t+1 \mid p)$ takes 1 under the condition by $q\left(n_{1} / t \mid p\right)$ and the probability function $\operatorname{Prob}\left(C_{1}(t)=n\right)$ for $p$ by $P(t, n \mid p)$. The master equation for $P(t, n \mid p)$ is

$$
\begin{aligned}
P(t+1, n \mid p) & =q((n-1) / t \mid p) \cdot P(t, n-1 \mid p) \\
& +(1-q(n / t \mid p)) \cdot P(t, n \mid p) .
\end{aligned}
$$

The expected value of $Z(t \mid p)=\frac{1}{t} C_{1}(t)$ is then estimated as

$$
\mathrm{E}(Z(t \mid p))=\sum_{n=0}^{t} P(t, n \mid p) \cdot \frac{n}{t}
$$


We are interested in the limit value of $Z(t \mid p)$ as $t \rightarrow \infty$, which we denote as $z$ :

$$
z \equiv \lim _{t \rightarrow \infty} Z(t \mid p)
$$

In the one-peak phase, $Z(t \mid p)$ always converges to $E(Z(t \mid p))$ in the limit, which we denote as $z_{+}$. In the two-peak phase, in addition to $z_{+}, Z(t \mid p)$ converges to a value smaller than half, which we denote as $z_{-}$, with some positive probability. It is a probabilistic process and one cannot predict to which fixed point $Z(t \mid p)$ converges. To determine the threshold value $p_{c}$ between these phases and the limit value $z_{ \pm}$, one needs to solve the following self-consistent equation: ${ }^{18}$

$$
z=q(z \mid p)=(1-p)+p \cdot q_{h}(t, t \cdot z)
$$

Given $p$, if there is only one solution, it is $z_{+}$and the system is in the one-peak phase. The convergence exponent $\gamma$ is obtained by estimating the slope of $q(z \mid p)$ at $z=z_{+}{ }^{18,27}$ If there are three solutions, which we denote as $z_{1}<z_{u}<z_{2}, z_{1}\left(z_{2}\right)$ corresponds to $z_{-}\left(z_{+}\right)$. The middle solution $z_{u}$ is an unstable state and $Z(t \mid p)$ departs from $z_{u}$ as $t$ increases. The method gives the rigorous results for $z$ and $\gamma$ where $q(z \mid p)$ is given as smooth function of $z$.

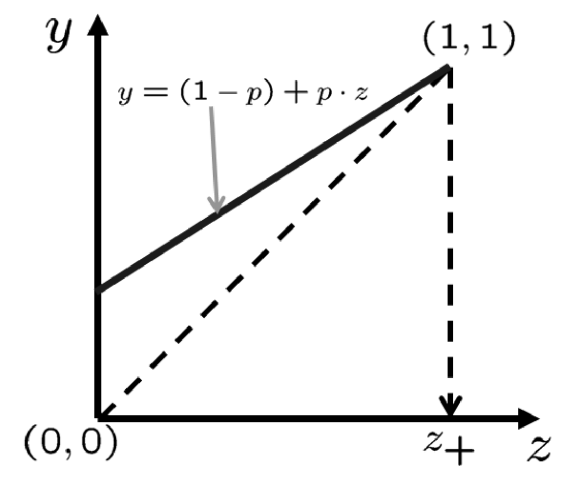

Fig. 4. Schematic view of the self-consistent equation $z=q(z \mid p)=(1-p)+p \cdot q_{h}(t, t \cdot z)$ for the system of analog herders : $q_{h}(t, t \cdot z)=z \cdot(z, q(z \mid p))$ connects $(0,1-p)$ and $(1,1)$ by a direct line. There is only one stable solution $z_{+}$at $z=1$ for $p<1$.

Figure 4 shows the case of analog herders and $q(z \mid p)=(1-p)+p \cdot q_{h}(t, t \cdot z)$ with $q_{h}(t, t \cdot z)=z \cdot{ }^{25}$ As one can easily see, for any value of $p<1$, there is only one stable solution $z_{+}$at $z=1$. The system is in the one-peak phase and $Z(t \mid p)$ always converges to $z_{+}=1$ for $p<1$. As the independent voter's probability of correct choice $q$ is $100 \%$, that of herders is estimated as 1 by $\left(z_{+}-(1-p) \cdot 1\right) / p$. Even in the worst limit $p \rightarrow 1$, the system of analog 
herders can take the probability of correct choice to one.

\subsection{Transition ratio $p_{c}(r)$ for cases $r \in\{C, M\}$}

Table III. Transition ratio $p_{c}$ of the voting (average herders) model. We determine $p_{c}$ using the condition that the self-consistent equation (7) has three or more solutions for $p>p_{c}$.

\begin{tabular}{ccccc}
\hline EXP. & $r$ & $p_{c}(r)$ & $r$ & $p_{c}(r)$ \\
\hline I & $C$ & $86.0 \%$ & $M$ & $95.7 \%$ \\
II & $C$ & $86.5 \%$ & $M$ & $96.7 \%$ \\
\hline
\end{tabular}

We introduce an average herders model where $q_{h}(t, t \cdot z)$ is given by linear extrapolation of the values $q_{h}\left(t, n_{1} \mid r\right)$ in equation (5). In our previous work, ${ }^{16}$ we model the behavior of herders by the following functional form with two parameters $a$ and $\lambda$ :

$$
\frac{1}{2}\left(a \tanh \left(\lambda\left(n_{1} / t-1 / 2\right)\right)+1\right) \text {. }
$$

However, the fitted result by the standard maximum likelihood estimation cannot capture the behavior of herders in the crucial region $n_{1} / t \sim 1 / 2$. We adopt the above linear extrapolated $q_{h}\left(t, n_{1} \mid r\right)$ for $q_{h}(t, t \cdot z)$ and solve the self-consistent equation (7). We determine $p_{c}(r)$ for cases $r \in\{C, M\}$ by the condition that the self-consistent equation has three or more solutions. We summarize the results in Table III. In case $C, p_{c}(C)$ is from $86.0 \%$ (EXP-I) to $86.5 \%$ (EXPII). In case $M, p_{c}(M)$ is from $95.7 \%$ (EXP-I) to $96.7 \%$ (EXP-II). However, these estimates depend on the behavior of $q_{h}\left(t, n_{1}\right)$ near $n_{1} / t=1 / 2$ where the estimate errors are big. We can at most say that $p_{c}(M)>p_{c}(C)$.

\subsection{Herder's probability of correct choice}

We estimate the probability of correct choice by a herder as a function of $p \cdot{ }^{29}$ As for the voting model, it can be estimated using the expectation value of $Z(t \mid p)$ as

$$
\mathrm{E}((Z(t \mid p)-(1-p) \cdot 1) / p)
$$

For the experimental data, we take the average of $(Z(i \mid r)-(1-p(i)) \cdot 1) / p(i)$ over the samples in $I$ (No.):

$$
\frac{1}{\mid I(\text { No. }) \mid} \sum_{i \in I \text { (No.) }}(Z(i \mid r)-(1-p(i)) \cdot 1) / p(i)
$$

We plot the results in Figure 5. The experimental results show that the probability of correct choice in case $C$ is better than that in case $M$ except for the samples in $I(6)$. As system size $T$ 


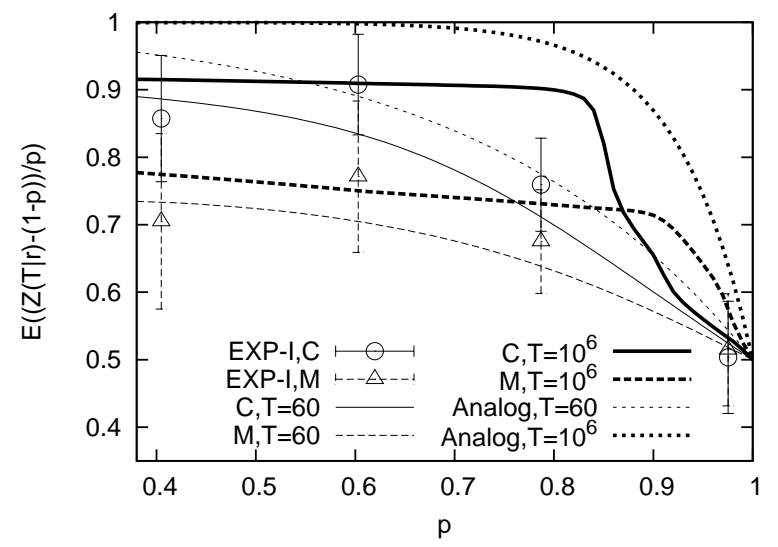

Fig. 5. Plot of herders' probability of correct choice, $(\mathrm{E}(Z(T \mid r))-(1-p)) / p$ vs. $p$, for the voting model. Symbol $\circ(\triangle)$ indicates the experimental data for the four bins $I(6), I(7), I(8)$, and $I(9)$ in Table II for case $C(M)$. The lines show the results of the stochastic model with system size $T=60, r=C$ (thin solid); $T=60, r=M$ (thin dashed); $10^{6}, r=C$ (thick solid); and $10^{6}, r=M$ (thick dashed). We also plot the result of the stochastic model for analog herders $q_{h}\left(t, n_{1}\right)=n_{1} / t$ with $T=60$ (thin dotted) and $10^{6}$ (thick dotted).

increases, for $p<p_{c}(C)$, the probability of correct choice in case $C$ remains better than that in case $M$. However, the maximum value of $q_{h}\left(t, n_{1} \mid C\right)$ is about 0.9 and the probability of correct choice saturates at the value for $p<p_{c}(C)$. As $p$ exceeds $p_{c}(C)$, the probability of correct choice in case $C$ rapidly decreases and dips below that in case $M$. From the information cascade transition, herders' probability of correct choice is much lowered and this results in the poor performance. In contrast, the poor performance of herders in case $M$ for $p<p_{c}(C)$ comes from the saturation of $q_{h}\left(t, n_{1} \mid M\right)$ at $n_{1} / t=3 / 4$. From the saturation, the probability of correct choice cannot reach the high value. For comparison, we show the results of the optimal system of analog herders with $T=60$ and $10^{6}$. In the thermodynamic limit, the probability of correct choice converges to one for $p<1$.

\section{Conclusions}

Social influence, which here is restricted only to information regarding the choices of others, yields inaccuracy in the majority choice. If a herder receives summary statistics $\left\{C_{A}, C_{B}\right\}$ and the payoff for the correct choice is constant, he strongly tends to copy the majority. The correct information given by independent voters is buried below the herd and the majority choice does not necessarily teach us the correct one if herders' proportion exceeds $p_{c}(C){ }^{16}$ When the return is set to be proportional to multipliers $\left\{M_{A}, M_{B}\right\}$ that are inversely proportional to summary statistics $\left\{C_{A}, C_{B}\right\}$, the situation is a zero-sum game between a herder and other previous subjects who have set the multipliers. The optimal max-min strategy is that of 
analog herders who choose $\alpha \in\{A, B\}$ with probability proportional to $C_{\alpha}$. Furthermore, the system of analog herders with $q=1$ maximizes the probability of the correct choice for any value of $p$ in the thermodynamic limit. Even in limit $p \rightarrow 1$, only the system can take the probability of correct choice to one.

We performed a laboratory experiment to study herders' behavior under the influence of multipliers $\left\{M_{A}, M_{B}\right\}$. We showed that they collectively behave almost as analog herders for $4 / 3 \leq m \leq 4$, where $m$ is the multiplier. Outside the region, herders' copy probability $q_{h}\left(t, n_{1} \mid M\right)$ saturates at about $3 / 4$ for $n_{1} / t \geq 3 / 4$ and it deviates from that of analog herders', $q_{h}\left(t, n_{1}\right)=n_{1} / t$. As a result, the probability of correct choice by a herder cannot reach a high value as compared to in the system of analog herders.

The system size and number of samples in our experiment are very limited, and thus it is difficult to estimate $p_{c}$ precisely. More importantly, in the estimation of $p$, we assume herder's $q$ is $50 \%$. It is the precondition of the experiment and we eliminate data which does not fulfill the condition. However, the procedure does not assure the precondition. In order to estimate $p$ more precisely and check the precondition, it is necessary to improve the experimental design or the data analysis procedure. In addition, in our experimental setup, the subjects have to choose between A and B. In addition, in our analysis of the experimental data, we only observe the average behavior of many herders. An interesting problem is whether a herder can adopt the max-min strategy at the individual level or only the average herder can do it. In order to clarify this, one good way is to permit people to divide their choice and vote fractionally. If the fraction voted by a subject is proportional to the summary statistic of previous subjects' choices, it suggests that the subject can adopt the max-min strategy at the individual level. We think that a more extensive experimental study of the system and of the related systems deserves further attention. ${ }^{30}$ Such experimental studies should provide new approach to econophysics ${ }^{24,31-35}$ and socio-physics. ${ }^{36}$

\section{Acknowledgment}

We thank Yosuke Irie and Ruokang Han for their assistance in performing the experiment. This work was supported by Grant-in-Aid for Challenging Exploratory Research 25610109. 


\section{References}

1) D. Austen-Smith and J. S. Banks: Am. Pol. Sci. Rev. 90 (1996) 34.

2) J. Surowiecki: The Wisdom of Crowds (Doubleday, New York, 2004).

3) S. E. Page: The Difference (Princeton University Press, Princeton, 2008).

4) P. Miller: The Smart Swarm (Avery Trade, London, 2011).

5) J. Lorenz, H. Rauhut, F. Schweitzer, and D. Helbing: Proc. Natl. Acad. Sci. (USA) 108 (2011) 9020.

6) L. Rendell, R. Boyd, D. Cownden, M. Enquist, K. Eriksson, M. W. Feldman, L. Fogarty, S. Ghirlanda, T. Lillicrap, and K. N. Laland: Science 328 (2010) 208.

7) L. Rendell, L. Fogarty, W. Hoppitt, T. Morgan, M. Webster, and K. Laland: Trends Cogn. Sci. 15 (2011) 68.

8) S. Bikhchandani, D. Hirshleifer, and I. Welch: J. Polit. Econ. 100 (1992) 992.

9) L. R. Anderson and C. A. Holt: Am. Econ. Rev. 87 (1997) 847.

10) D. Kubler and G. Weizsacker: Rev. Econ. Stud. 71 (2004) 425.

11) J. Goeree, T. R. Palfrey, B. W. Rogers, and R. D. McKelvey: Rev. Econ. Stud. 74 (2007) 733.

12) I. H. Lee: J. Econ. Theory 61 (1993) 395.

13) A. Devenow and I. Welch: Euro. Econ. Rev. 40 (1996) 603.

14) D. J. Watts: Proc. Natl. Acad. Sci. (USA) 99 (2002) 5766.

15) B. Latané: Am. Psychol. 36 (1981) 343.

16) S. Mori, M. Hisakado, and T. Takahashi: Phys. Rev. E 86 (2012) 026109.

17) M. Hisakado and S. Mori: J. Phys. A 44 (2011) 275204.

18) M. Hisakado and S. Mori: J. Phys. A 45 (2012) 345002.

19) D. Hausch, V. S. Y. Lo, and W. Ziemba: Efficiency of Racetrack Betting Markets (World Scientific, Singapore, 2008).

20) M. Ali: J.Pol. Econ. 85 (1977) 803.

21) J. Wolfers and E. Zitzewitz: J. Econ. Persp. 18 (2004) 107.

22) C. Manski: Econ.Lett. 91 (2006) 425.

23) J. V. Neumann and O.Morgenstern: Theory of Games and Economic Behavior (Princeton University Press, Princeton, 1944). 
24) S. Mori and M. Hisakado: J. Phys. Soc. Jpn. 79 (2010) 034001.

25) M. Hisakado and S. Mori: J. Phys. A 43 (2010) 315207.

26) H. F. Knight: Risk, Uncertainty, and Profit (Hart, Schaffner and Marx, Boston, 1921).

27) S. Hod and U. Keshet: Phys. Rev. E 70 (2004) 015104.

28) D. Sumpter and S. C. Pratt: Phil. Trans. R. Soc. B364 (2009) 743.

29) P. Curty and M. Marsili: J. Stat. Mech. 2006 (2006) P03013.

30) M. J. Salganik, P. S. Dodds, and D. Watts: Science 311 (2006) 854.

31) R. N. Mantegna and H. E. Stanley: Introduction to Econophysics: Correlations and Complexity in Finance (Cambridge University Press, Cambridge, 2007).

32) T. Lux: Econ. J. 105 (1995) 881.

33) A. Kirman: Q. J. Econ. 108 (1993) 137.

34) R. Cont and J. Bouchaud: Macroecon. Dynam. 4 (2000) 170.

35) J. González-Avella, V. Eguíluz, M. Marsili, F. Vega-Redondo, and M. S. Miguel: PLoS One 6 (2011) e20207.

36) S. Galam: Int. J. Mod. Phys. C 19 (2008) 409.

\section{Appendix A: Additional information about the Experiment}

In EXP-I, 120 subjects were recruited from the Literature Department of Hokkaido University. We made two groups of about sixty subjects and the subjects in each group answered 120 questions one by one. Because of the capacity of the laboratory, we could not perform the whole experiment at a time. We divide the subjects of each group into five sub-groups of about 12 subjects. In one session, subjects in a sub-group sequentially answered the questions. After five sessions we have gathered the data from all the subjects in a group.

Subjects were paid in cash upon being released from the session. There was a 500 yen (about 5 dollars) participation fee and additional rewards that were proportional to the number of points gained. In cases $O$ and $C$, one correct choice was worth two points, and one point was worth one yen (about one cents). In case $M$, one correct choice was worth the multiplier itself. In the main text, we treat case $M$ as zero-sum game. Considering the participation fee, we can regard it as constant sum game, which is equivalent to a zero-sum game. As for EXP-II, detailed information can be found in. ${ }^{16}$ 


\section{Voting Experiment}

\section{Payoff Odds Info.}

Up to now 9 subjects have answered.

Their choices are given as multipliers as folows.

If your choice is true, the points earned is the multiplier.

Even if a choice with a large mutiplier is more likely to be wrong, it is rational to choose it with the objective of expected return. Please choose.

Q.30:Which composer is famous for Symphonie No. 6 Pathetique?

A : Beethoven B : Tchaikovsky

$\times 1.1 \times 5$

Fig. B.1. Snapshot of the screen for case $M$. Multipliers $\left\{M_{A}, M_{B}\right\}$ are given in the second row in the box.

\section{Appendix B: Experimental procedure}

We explain the experimental procedure in EXP-I in detail. All the subjects in a sub-group entered the laboratory and sat in the partitioned spaces. Using slides, we showed subjects how the experiment would proceed. We explained that we were studying how their choices were affected by the choices of others. In particular, we emphasized that social information was realistic information calculated from the choices of previous subjects. Through the slides, we also explained how to calculate multipliers $\left\{M_{A}, M_{B}\right\}$ in case $M$, with a concrete example.

After the explanation, the subjects logged into the experiment web site using their IDs and started to answer the questions. Interaction between subjects was permitted only through the social information given by the experiment server. A question was chosen by the experiment server and displayed on the monitor. First, subjects answered the fist half of the 120 questions $i \in\{1,2, \cdots, 60\}$ using only their own knowledge $(r=O)$. After answering all the sixty questions in case $O$, the subjects answered the same 60 questions in case $C$. Finally, the subjects answered the same questions in case $M$. In each case, the experiment server chose a question among the sixty questions at random that was not served to the other subjects at the time. Otherwise, we cannot give correct social information to the $t+1$-th subject from all previous $t$ subjects. After a five-minute interval, we repeated the same procedure so that the subjects answered all 120 questions.

Figure $\mathrm{B} \cdot 1$ shows the experience of the subjects in case $M$. In the example covered in the figure, already nine subjects have answered question 30. The multipliers are given in 
the second row along with the number of subjects who answered the question. Only one subject among nine has chosen A and the remaining eight subjects have chosen B. Multiplier $M_{A}\left(M_{B}\right)$ is calculated as $10 /(1+1)=5(10 /(8+1)=1.1)$. The multipliers are rounded off to one decimal place.

In EXP-II, the experience of the subjects is almost the same as in EXP-I. ${ }^{16}$ The difference lies in how the experiment proceeds. In EXP-II, each subject answered each question from case $O$ to case $M$. After that, the experiment server chose another question. The process continues until the subject has answered all questions. The subjects were likely to easily remember the answers for the earlier cases with different social information and be careful in choosing answers in the later cases. In order to exclude such an effect, we changed the system to that in EXP-I.

\section{Appendix C: Contorollability of the difficulty level of a question}

We have used the same 120 questions in EXP-I and EXP-II. For the selection process, please refer to our previous paper. ${ }^{16}$ Here, we study whether the difficulty of a question is an inherent property or not. For this purpose, we compare the percentage of correct answers to each question in case $\mathrm{O}$ in Group $\mathrm{A}$ and in Group B. It is defined for Group $\mathrm{A}$ as $Z(i \mid O)=$ $\sum_{s=1}^{T_{i}} X(i, s \mid O) / T_{i}$ and for Group B as $Z(i+120 \mid O)=\sum_{s=1}^{T_{i+120}} X(i+120, s \mid O) / T_{i+120}$. We show the scatter plot $\{Z(i \mid O), Z(i+120 \mid O)\}$ in Figure $\mathrm{C} \cdot 1$.

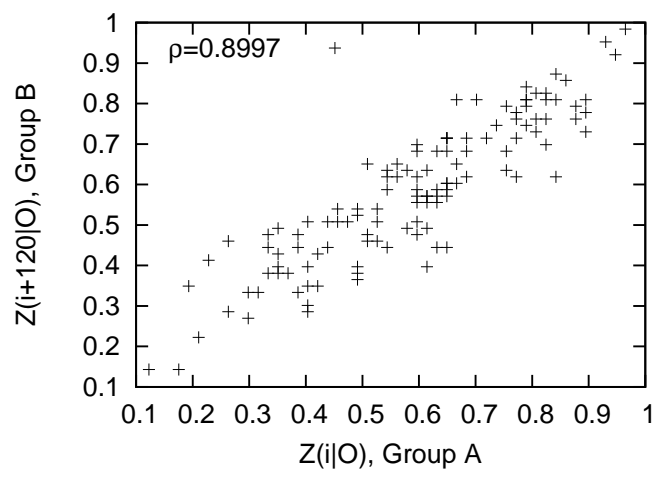

Fig. C.1. Scatter plots of $Z(i \mid O)$ vs. $Z(i+120 \mid O)$ in EXP-I. Pearson's correlation coefficient $\rho$ is 0.8997 .

As one can clearly see the distribution almost on the diagonal line, we can infer that there is a strong correlation. Pearson's correlation coefficient $\rho$ is about 0.90 . In EXP-II, we observe the same feature and $\rho$ is about 0.82 . The strong correlation means that if a question is difficult (easy) for the subjects in a group, it would also be difficult (easy) for the subjects 
in the other group. The system sizes in our experiments are very limited and there remains some fluctuation in the estimation of $Z(i \mid O)$, but it will disappear for a large system. We can control the difficulty levels of the questions in the experiment and study the response of a subject under controlability. This aspect is important when one makes some prediction based on the results presented in this paper.

\section{Appendix D: Uniqueness of the analog herders system}

In the main text, we show that the system of analog herders maximizes the probability of correct choice for $p<1$ and can take it to one for any $p<1$. Here, we show that only the system of analog herders can do it.

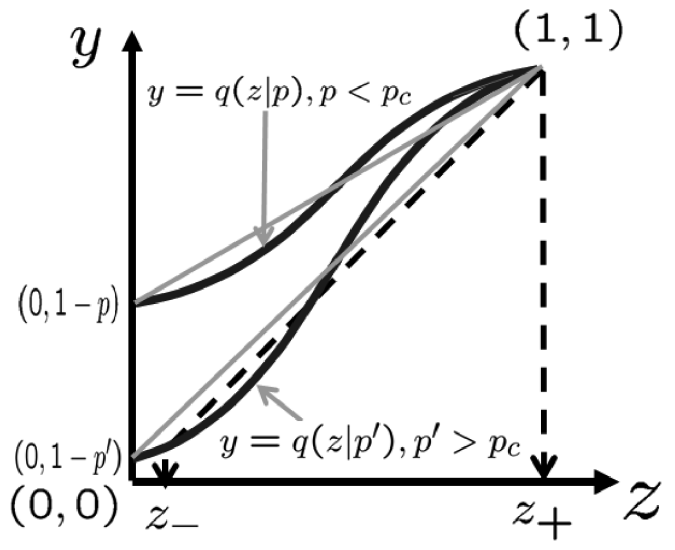

Fig. D-1. Schematic view of the self-consistent equation $z=q(z \mid p)=(1-p)+p \cdot q_{h}(t, t \cdot z)$ with general $q_{h}(t, t \cdot z) \cdot(z, q(z \mid p))$ connects $(0,1-p)$ and $(1,1)$ by a continuous curve. As $z_{+}=1$ is a stable solution, $\frac{q(z \mid p)}{d z}$ at $z=1$ is one or less. If $q_{h}(t, t \cdot z)$ deviates from $z$, for $p^{\prime}>p_{c}$, in addition to the stable solution $z_{+}$at $z=1$, there is at least one stable solution $z_{-}$for $z<1$.

As the system with analog herders assures that the probability of correct choice is one for any $p<1$, the self-consistent equation for the system considered must have only one stable solution $z_{+}$at $z=1$. If the equation has more than one stable solution and the probability of convergence to solutions less than one is finite, the probability of correct choice cannot take one. The self-consistent equation has a solution $z_{+}$at $z=1, q_{h}(t, t \cdot z)$ must take $1(0)$ at $z=1(0)$. In addition, as $z_{+}$is stable, the slope of $q(z \mid p)$ at $z=1$ is one or less. The curve $(z, q(z))$ connects $(0,(1-p))$ and $(1,1)$ as in Figure D.1. The curve of the system of analog herders connects the two points by a direct line. If $q_{h}(t, t \cdot z)$ deviates from $z$, the curve between the two points is rippling above and below the direct line. Then, one can see that there is some 
threshold value $p_{c}<1$, where for $p>p_{c}$, the curve has more than three intersections with the diagonal line $y=z$. In this case, in addition to the stable solution $z_{+}$, there exists another stable solution $z_{-}$less than one. The probability of correct choice becomes less than one and the statement is proved.

\section{Appendix E: Exponent $\gamma$}
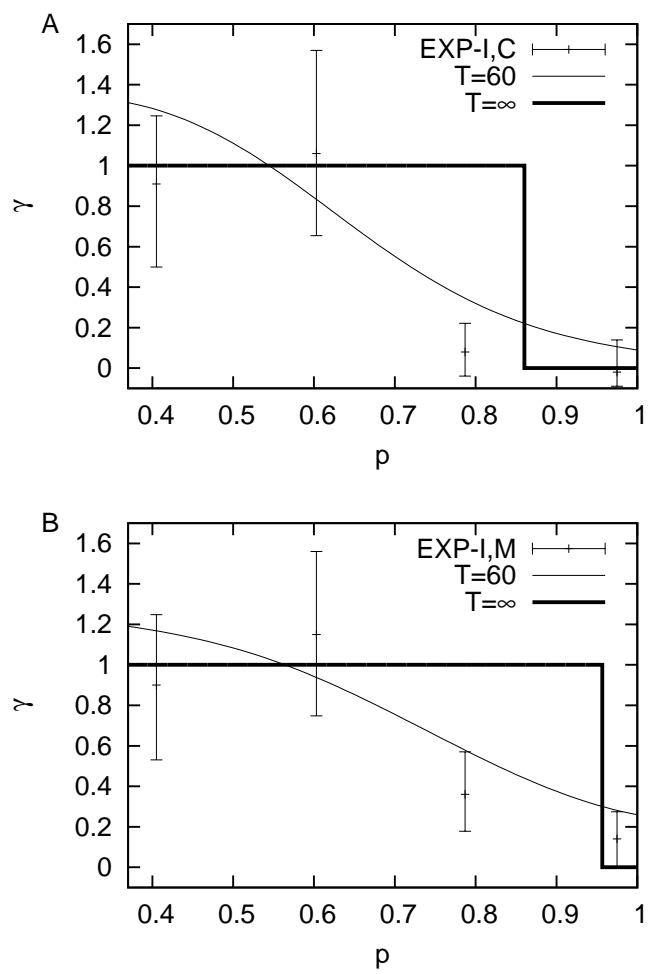

Fig. E.1. Plot of $\gamma$ vs. $p$. We plot the results of the average herders model for EXP-I for (A) Case $C$ and (B) Case M. Symbol (o) denotes $\gamma \mathrm{s}$ vs. $p_{\text {avg }}$ in EXP-I, which are estimated in Figure 2. The lines show the results of the stochastic model with system size $T=60$ (thin solid) and $T=\infty$ (thick solid).

In order to check the validity of the stochastic model for cases $r \in\{C, M\}$, we study the converge exponent $\gamma$. We solve the master equation (6) recursively and obtain $P(t, n \mid p)$ for $t \leq T=60$ for EXP-I. We estimate the convergence exponent $\gamma$ from the slope of $\operatorname{Var}(Z(t \mid p))$ as

$$
\gamma=\log \frac{\operatorname{Var}(Z(T-\Delta T \mid p))}{\operatorname{Var}(Z(T \mid p))} / \log \frac{T}{T-\Delta T} .
$$

We take $\Delta T=50$ to match the analysis of the experimental data in Figures $2 \mathrm{~A}$ and $\mathrm{B}$. In order to give the error bar of $\gamma$ for the experimental results, we adopted the voting model to simulate the system and estimate the $95 \%$ confidence interval. ${ }^{16}$ For $T=\infty$ (thermodynamic 
limit), we estimate the gradient $q^{\prime}\left(z_{+} \mid p\right)$ of $q(z \mid p)$ at $z=z_{+}$and use the formula $\gamma=\operatorname{Min}(1,2-$ $\left.2 \cdot q^{\prime}\left(z_{+} \mid p\right)\right){ }^{18}$ The results are summarized in Figure $\mathrm{E} \cdot 1$ for (A) case $C$ and (B) case $M$. For $T=60$, the model describes the experimental results well. In the limit $T \rightarrow \infty, \gamma$ monotonically decreases from 1 to 0 . 